\title{
Proceso de aprendizaje agroecológico desde la práctica de la meliponicultura: una experiencia de campesinos en Santa Lucía, Nicaragua
}

\section{Agroecological learning process from the practice of meliponiculture: an experience of peasant farmers in Santa Lucia, Nicaragua}

\section{Yorlis Gabriela Luna Delgado ${ }^{1}$, Jader Antonio Angulo Sobalvarro ${ }^{2}$}

${ }^{1}$ MSc. En Ciencias Matemáticas, Número https://orcid.org/0000-0002-3879-8252 ylunadel282003@yahoo.com Instituto Nicaraguense de Tecnología Agropecuario (INTA)

2 Ing. Agrónomo, Número https://orcid.org/0000-0003-3276-8869 jadersobalvarro83@gmail.com

Instituto Nicaraguense de Tecnología Agropecuario (INTA)

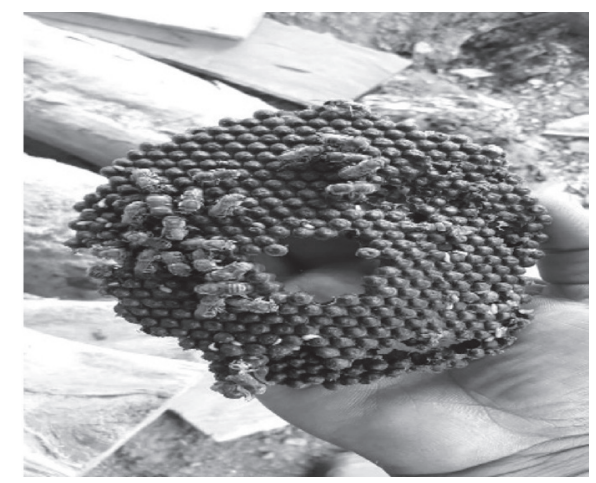

\section{RESUMEN}

La agroecología como práctica, ciencia y política, es la única alternativa para crear sostenibilidad, resiliencia y una nueva forma de vida que resignifique los territorios campesinos. Por eso, para preservar la vida en el planeta son urgente acciones y estrategias que conlleven a que cada vez un mayor número de familias agricultoras asuman la agroecología como forma de producir y de vivir. Esto es un proceso de aprendizaje donde intervienen diferentes mediadores pedagógicos que facilitan o retrasan el propio aprendizaje. En el presente trabajo, analizamos el proceso de aprendizaje de la agroecología desde la práctica de la meliponicultura en Santa Lucia, Boaco, donde a partir de una serie de intercambios de campesino a campesino en meliponicultura se ha logrado revitalizar el movimiento agroecológico. Alcanzando en pocos meses 50 nuevas familias meliponicultores, de ellos 25 nuevos productores con prácticas agroecológicas; con 115 cajas y 25 troncos con los cuales están rescatando cinco tipos de abejas nativas. En este proceso los mediadores pedagógicos han sido la actividad con meliponinos, la relación con promotores agroecológico de experiencia, la cultura local, el liderazgo y fortaleza organizativas territoriales.

Palabras clave: meliponinos, aprendizaje, mediación, agroecología.

\section{ABSTRACT}

Agroecology - as practice, science, and politics, is the only alternative for creating sustainability, resilience and a new form of life that resignifies peasant territories. In order to preserve life on the planet, actions and strategies are needed that lead an ever-greater number of farm families to take on agroecology as a form of production and form of life. This is a learning process in which distinct pedagogical mediators intervene, facilitating or obstructing learning. In this article, we analyze the learning process of agroecology from the practice of meliponiculture in Santa Lucia, Boaco, Nicaragua, where, after a series of peasant to peasant exchanges in meliponiculture, the agroecological movement has been revitalized. Between January and June 2018, 50 new families began producing melipona bees, and 25 of these families employ agroecological practices. In total, 115 boxes and 25 tree trunks were producing five different species melipona bees at the time of field work in June 2019. In this process, pedagogical mediators have included the practical activity with meliponinos, the relationship with experiences agroecological promotors, local culture, and the territorial leadership and organizational strength of producer families.

Keywords: meliponinos, learning, mediations, agroecology.
Recibido: 8 de agosto del 2019 Aceptado:2 de octubre del 2019

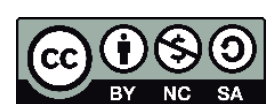

Los artículos de la revista La Calera de la Universidad Nacional Agraria, Nicaragua, se comparten bajo términos de la licencia Creative Commons: Reconocimiento, No Comercial, Compartir Igual. Las autorizaciones adicionales a las aquí delimitadas se pueden obtener en el correo edgardo.jimenez@ci.una.edu.ni

(C) Copyright 2019. Universidad Nacional Agraria 
$\mathrm{N}$ icaragua tiene una alta vulnerabilidad frente al cambio climático, por eso es urgente construir estrategias que permitan mitigar sus efectos y crear resiliencias a nivel de paisajes. Para lo cual, es indispensable analizar las experiencias exitosas en el corto y mediano plazo de avances en la transición y masificación agroecológica en los diferentes territorios del país, ya que para las fincas y paisajes agroecológicos son sostenibles y resilientes al cambio climático (Machin, Jaime, Lozano y Rosset, 2010).

La agroecología es una alternativa frente al cambio climático, en Nicaragua, sin embargo, faltan el estudio de las metodologías y estrategias que permitan llevar la agroecología a escala, de ahí la necesidad de analizar las experiencias exitosas de transición agroecológica y los factores que facilitan y limitan el escalonamiento agroecológico. Este estudio pretende aportar elementos de análisis desde el aprendizaje de la agroecología en Santa Lucia, Boaco para la construcción de estrategias para la masificación agroecológica.

La agroecología implica un proceso de aprendizaje social e histórico, que tiene dimensiones ambientales, organizativas y sociales (Sevilla, 2011). Para llevar la agroecología a escala son claves los procesos de aprendizaje (Mier et al., 2018); los cuales implican apropiación de nuevas formas sociohistóricas, cambios en el pensar, sentir y actuar que se materializan en las actividades concretas que los seres humanos realizan a nivel individual y colectivo (Luna, 2012).

Estos "cambios de mentalidad" en los territorios agrarios es un cambio de cultura productiva, ya que, por la naturaleza histórica y social del ser humano, la cultura y la naturaleza forman parte de un mismo proceso dinámico y continuo. Cada cultura y cada momento histórico son interdependientes para producir los esquemas y dinámicas específicas de "manejo" de la naturaleza (Lazos, 2009).

Por tanto, el paisaje es el resultado de la acción humana, de la apropiación e interacciones culturales; y "se torna significativo en la práctica, en lo cotidiano, entre actores y sus conocimientos" (Lazos, 2009). De ahí, que es en las acciones cotidianas de la vida que se reconstruyen y resignifican diariamente los territorios (Escobar, 2015).

En la cotidianidad los paisajes se reconfiguran, con aprendizajes; que en sí mismos son procesos mediados. Entendemos a los mediadores como todos los factores que facilitan o inhiben el proceso de aprendizaje, parte intrínseca del propio aprendizaje (Castellanos, Silverio, Reinoso y García, 2002) y que describen y configuran su esencia (Luna, 2012).
Algunos de los mediadores más desarrollados teóricamente son la interacción directa con el otro y la actividad. La actividad definida como todas las formas de actuación del ser humano ya sean internas o externas, que están dirigidas a la solución de tareas concretas y que están reguladas de manera consciente (Talizina, 2000).

El trabajo con las abejas nativas o meliponinos crea nuevos valores, resignifica los conocimientos, reconstruye, revaloriza y reafirma las identidades, crea orgullo y nuevos sentidos de vida (Genaro y Lóriga, 2018). La meliponicultura permite crear nuevas relaciones entre las abejas y los seres humanos a mediano y largo plazo, relaciones que van más allá de lo utilitario, económico y productivista (Chan, Anilú, Vera, Aldasoro y Sotelo. 2019).

Los meliponinos son los principales polinizadores en los ecosistemas de Mesoamérica, su servicio ecológico es más eficiente por sus profundas relaciones con redes mutualistas planta-polinizador en los diferentes tipos de ecosistemas (Ayala y Ortega, 2018; Brosi, Daily, Shih, Oviedo y Durán, 2008). En Nicaragua hay una gran diversidad de abejas nativas; algunas de estas abejas han sido cuidadas y cosechadas por los pueblos desde tiempos precolombinos (Guzmán, Balboa, Vandame, Albores y González-Acereto; 2011), por lo que forman parte de los saberes contemporáneos, la memoria, la cultura e identidad de los pueblos y el patrimonio biocultural de los pueblos (Aldasoro, 2012).

Las abejas nativas sin aguijón se encuentran en peligro de extinción junto a todos los polinizadores del mundo, por el modelo dominante de la revolución verde causante del cambio climático, de la contaminación por residuos de agroquímicos en los suelos, el avance de la frontera agrícola, la contaminación del agua y aire (Rodríguez, McLaughlin, y Pennock, 2018); la pérdida de biodiversidad y fragmentación del hábitat. Con la extinción de las abejas, estaría en peligro la supervivencia humana y la reproducción de todas las formas de vida. La única alternativa para rescatar las abejas nativas es la agroecología; las prácticas agroecológicas favorecen el establecimiento de dinámica poblacionales en las abejas, que permiten la permanencia de las poblaciones en el tiempo (Perfecto y Vandermeer, 2012).

La matriz agrícola permite el movimiento de especies entre fragmentos de parches naturales y fincas que conforman los paisajes; las forma de manejos influyen directamente en la anidación y forrajeo; condiciones básicas para garantizar el desarrollo de las poblaciones de abejas en fragmentos de parches naturales; cuando se fragmentados los espacios, se amplían la distancia entre el nido y la 
alimentación por lo que las poblaciones se disgregan y se hace más difícil su supervivencia en el tiempo (Meléndez, Ayala, Delfín y Manrique, 2017).

El municipio de Santa Lucia, Departamento de Boaco en Nicaragua fue uno de los lugares donde proliferó el Programa Campesino a Campesino para la Agricultura Sostenible en Nicaragua (PCAC), con una buena proporción de fincas y familias locales siendo agroecológicas. Por tal razón, en el paisaje de Santa Lucía es notable las curvas a nivel, barreras vivas y obras de conservación de suelo.

PCAC fue impulsado por la Unión Nacional de Agricultores y Ganaderos (UNAG). Su metodología se caracteriza por ser horizontal, basada en los principios de la educación popular, el conocimiento local, el dialogo de saberes entre pares y su principal herramienta es el intercambio campesino (Machín et al., 2010). Para el año 2000, este programa llegó a tener al $28 \%$ de las familias rurales nicaragüenses involucradas (Holt-Giménez, 2008), siendo un referente para el movimiento agroecológico en una gran parte de los territorios del país.

A partir del 2008, el Instituto Nicaragüense de Tecnología Agropecuaria (INTA), dentro de una política pública de promotoría agroecológica y fincas de innovación, retomó a una gran parte de productores del PCAC y acompañó la continuidad de los intercambios. A partir de enero del 2019, el INTA en articulación con la Alcaldía Municipal y los líderes territoriales, iniciaron un conjunto de capacitaciones en meliponicultura a productores del municipio en varias comunidades, algunos de ellos líderes históricos del PCAC.

A finales del año 2018 en distintos, talleres, giras de campo y capacitaciones algunos productores planteaban que tenían troncos con abejas nativas, pero desconocían como cosecharlas y que les interesaba incursionar en el rubro. A partir de ahí, una alianza territorial basada en instituciones de gobierno, Alcaldía Municipal, líderes locales, productores líderes y jóvenes del movimiento ambientalista, organizaron intercambios de conocimientos en meliponicultura.

En un periodo de enero a junio del 2019, se dinamizaron los intercambios de campesino a campesino en meliponicultura y más de 50 familias comenzaron a rescatar y producir estas abejas nativas; aproximadamente la mitad de las familias en este período iniciaron una transición hacia la agroecología en sus fincas. En el presente trabajo, realizaremos un estudio de caso, analizando el proceso de aprendizaje de la agroecología tomando como mediador pedagógico la práctica de la meliponicultura en el contexto histórico-cultural del territorio de Santa Lucia, Boaco.
Basado en la relevancia que tiene el CAC en las matrices agrícolas que conservan biodiversidad (Perfecto y Vandermeer, 2012), estas experiencias muestran casos en que las familias agroecológicas de experiencia en CAC son las que más se están apropiando de aprender y enseñar la meliponicultura, y a partir de sus liderazgos, otros productores se motivan en la meliponicultura. Mientras tanto cuando productores convencionales participan en intercambios CAC en meliponicultura, se motivan por cuidar a las abejas e inician procesos de transición agroecológica en sus fincas.

Por tal razón, en este estudio abordaremos una posible relación en dos direcciones por un lado para realizar las actividades de proteger, cuidar, rescatar y garantizar la continuidad de las poblaciones de abejas nativas en los paisajes es urgente la masificación de la agroecología (Guzmán, Balboa, Vandame, Albores y González-Acereto, 2011). Y en sentido contrario, para la masificación de la agroecología, son necesarios mediadores pedagógicos del proceso de aprendizaje agroecológico y la meliponicultura se revela como uno de esos mediadores pedagógicos; inmerso en la cultura y espiritualidad local (Mier et al., 2018).

La importancia de este trabajo radica en que hay muy pocos estudios sobre los meliponinos en Nicaragua, y los que hay están enfocados en las cadenas de valor y la economía familiar (Montenegro, 2013); muy pocos se enfocan desde su relación con la masificación y escalonamiento de la agroecología.

\section{MATERIALES Y MÉTODOS}

El municipio de Santa Lucia, Boaco, fue uno de los casos emblemáticos del PCAC en Nicaragua. Por esa razón, cuenta con una amplia red de promotores y líderes agroecológicos, con fincas modelos y productores aledaños, esta red ha sido sostenida por la voluntad, consciencia, la creatividad y el entusiasmo de las familias con apoyo de algunos aliados externos, como lo son el INTA, la Alcaldía Municipal, la Asociación de Jóvenes Cristianos, líderes religiosos locales, territoriales y políticos.

Santa Lucia, Boaco, forma parte del llamado Corredor Seco de Nicaragua. La zona se caracteriza por estar situada a una altura entre 500 y $570 \mathrm{msnm}$ y la temperatura anual promedio oscila entre $\operatorname{los} 25^{\circ}$ y $30^{\circ} \mathrm{C}$, la topografía es irregular (INIDE, 2008); en la parte alta e intermedia del municipio tiene un considerable número de parches naturales con bosques en regeneración y áreas agroforestales de café, con condiciones aptas para anidación y forrajeo de las meliponinos. Es un estudio mixto, 
con herramientas cuantitativas y de la investigación acción-participativa.

Se trabajó con un universo de 260 productores, la muestra fueron 50 productores, por la naturaleza del trabajo se utilizó un muestro no aleatorio por cuotas o accidental.

El trabajo se desarrolló con familias productoras en las comarcas Los Garcías, El Llanito, Los Rivas, Los Alvares y Las Mercedes. La metodología del trabajo fue un enfoque mixto, herramientas cualitativas, grupo focal, entrevistas y encuestas, con el objetivo de recabar información acerca de las unidades de análisis sociales, como motivaciones, intensiones, expectativas y experiencias; observación participante durante tres meses, revisión de informes técnicos, análisis de contenido; herramientas de la investigación acción participativa y de la estadística descriptiva. Se realizó triangulación de la información, y la combinación de los métodos inductivo-deductivo.

\section{RESULTADOS Y DISCUSIÓN}

La caracterización de los 260 productores participantes es: $10 \%$ eran productores innovadores líderes con una amplia experiencia en el PCAC, con fincas con sistemas productivos con 20 años de rediseño y trabajan activamente con el INTA, el $35 \%$ productores aledaños con entre dos y cuatro años de realizar algunas actividades de sustitución de insumos y reducción o eliminación de insumos químicos y el 55\% eran nuevos productores.

Las actividades realizadas de intercambio de conocimiento fueron trampeo de especies, cambio de tronco a cajas racionales, proceso de alimentación, inducción a las variedades de meliponinos y su importancia en la polinización, realización de trampas para mosca vinagrera, aspectos generales del manejo, cosecha y división de colmenas, identificación y biología de las abejas, ecotipos, condiciones ambientales y saberes tradicionales. En los primeros intercambios participó el productor Yoni Cortes, de la Asociación de Productores Agroecológicos de Santa Teresa, Carazo, quien compartió sus experiencias a 20 productores líderes; a partir de ahí, se han realizado 35 intercambios, con más de 260 productores de todo el municipio.

A partir de este proceso, 50 productores y sus familias iniciaron con la producción de meliponinos; en cinco comarcas: Los Garcías, El Llanito, Los Rivas, Los Alvares y Las Mercedes. Comarcas donde están la mayor parte de productores innovadores con amplia experiencia en el PCAC.A la fecha de realización de este trabajo tienen 115 cajas, 36 de jicote barcino (Melipona beecheii), 51 de mariolita (Tetragonisca angustula), 13 soncuan chele (Scaptotrigona pectoralis), 11 de tamagás (Cephalotrigona zexmeniae) y cuatro de chicopipe (Nanotrigona mellaria).

De los 50 nuevos meliponicultores, ocho son líderes históricos del PCAC, 17 son familias que ya han iniciado algunas prácticas agroecológicas en los últimos cuatro años y 25 son familias nuevas que iniciaron a realizar prácticas agroecológicas a partir de la meliponicultura.

Las cajas han sido elaboradas por los propios productores y con madera local de las fincas, están rescatando al jicote barcino, mariolita, soncuan chele, tamagás y chicopipe. Han realizado divisiones de colmenas y pase de tronco a cajas, para evitar saqueos de nidos silvestres. Asimismo, cada productor instaló trampas en sus fincas, para un total de más de 101 botellas trampas, de las cuales 26 ya funcionaron para capturar mariolitas. Los productores del proceso plantean: "Ha sido una nueva experiencia, conocí a las abejas pero nunca me había interesado por tenerlas en mi casa, ni por tenerlas en una caja, es la función que ellas desarrollan, en la polinización de los cultivos, eso me ha motivado... me ha ayudado a tener y compartir con algotras personas y tener nuevas amistades...vienen a mi casa y cuando miran que estoy recolectando las abejas nativas, la gente se emociona, les llama la atención cuando les explican, se han motivado, viera la gente se van encantada, vienen me invitan a ir a sus fincas a ayudarles... imagínese hasta donde he llegado, siento que me ha hecho cambios en la vida, además de eso, .... son ingresos" (O.V. García, comunicación personal, 17 julio, 2019).

El conocimiento de los procesos de polinización y sus interrelaciones con el paisaje es uno de los mayores cambios citados por los productores: "Uno de los mayores cambios o logros tenidos es el aprendizaje... Me he dado cuenta que la producción se mueve gracias a la polinización de las abejas, además que las abejas nos dan el alimento cada día" (O.V García, entrevista 12 junio, 2019).

"Me gusta que se pueden incluir mi esposa y mis hijos en la alimentación, me gusta mayor integración con la comunidad... Yo a mis abejas les llamo mis niñas, mis princesas, mis reinas porque ellas son parte de nuestra familia y las miramos como las niñas de la casa" (J.A. Angulo, comunicación personal, 14 de junio, 2019). Es otra manera de trabajar para la sostenibilidad familiar, de relacionarse con las abejas, observarlas, su trabajo diario desde las 5 de la mañana sin parar hasta el anochecer, la organización de las mismas, les hace sentir y pensar a las familias que deben ayudarles. J.A. Angulo ( comunicación personal, 2 de febrero, 2019) declaro: "Los abuelos me 
enseñaron como lo alimentaron, contando que en su época ellos tuvieron 10 troncos y las cuidaban alimentándola con dulce de caña a la par del tronco cuando no había comida y una panita de agua con chibolas o tronquitos de madera para que las abejas se pararan en las chibolitas o madera, para que las abejas bajaran a beber agua sin ahogarse, para que no fueran largo a buscar agua, mis abuelos dicen que las abejas criollas son de nuestra familia"

En el caso estudiado de Santa Lucia, "el otro" son las abejas meliponinos y los promotores agroecológicos líderes del PCAC. Y "la actividad" son todas las actividades que implican la crianza de las abejas nativas, la capturas, los traslados, los cuidos y manejos tecnológicos, y las prácticas agroecológicas en las fincas. Es decir, la meliponicultura en sí misma es un mediador del aprendizaje de la agroecología a nivel individual y colectivo.

"Es otra manera de ver la vida, es un aprendizaje que no tiene precio porque las abejas no tienen precio... Las abejas son defensoras de la vida... Me gusta la forma de trabajar de las abejas porque son sin aguijón, fácil de trabajar y la producción de miel es medicinal, y sobre todo que son de mis abuelos" (Y.F. Cortez, comunicación personal, 28 de enero, 2019). "Dios nos dio la naturaleza para cuidarla, respetarla, las abejitas son seres de Dios, es un regalo que nos dio" (F.A.ron, les piden ayuda en la captura, cambio de tronco a cajas e intercambio de experiencias Sobalvarro, comunicación y capacitaciones. personal, 28 de enero, 2019).

El $87 \%$ de los productores encuestados plantean que las meliponinos han sido un proceso de aprendizaje, que los ha motivado a observar más el paisaje, la floración, estar pendiente del uso de químicos en los alrededores, $\mathrm{y}$ a interesarse más en la diversificación de la finca para garantizar floración y alimentación para las meliponinos. Plantean que les gusta por el hecho que no tienen aguijón y ser de los abuelos. Asimismo, hay una integración de mujeres en un $22 \%$.

En los distintos momentos, los productores concluyeron que la meliponicultura "es una forma de vida, las meliponinos producen poco, pero es más una forma de cuidar las comunidades y las familias" (M.A. Angulo, 18 febrero, 2019). Un productor manifestó, "Yo comencé recogiendo un tronco viejo que estaba en la casa, luego me di cuenta de que en ese tronco todavía había abejitas, y cuando conocí la importancia de la polinización fui a rescatarlo y le pregunté a mi abuelita y ese tronco estaba con mi familia desde que mis abuelitos se casaron" (Y.F. Cortez, comunicación personal,10 de marzo, 2019).

El símbolo identitario inherente en la meliponicultura (por ser de los abuelos, bisabuelos y asociarlas a su propia identidad familiar); la facilidad, la mancedad de las abejas, el manejo cercano con las casas y el rápido aprendizaje ha motivado a los productores convencionales por las abejas nativas (INTA, 2019) y con ellos se han motivándose en los procesos de experimentación, prueba y error en uso de bioinsumos, reducción y eliminación de agroquímicos, diversificación de fincas, y de todas las prácticas y principios de la agroecología. Iniciando una especie de transición y aprendizaje agroecológico mediado por las meliponinos y los productores de experiencia del PCAC.

Estos productores agroecológicos de experiencia fueron los que más rápidamente se apropiaron de la tarea de aprender y enseñar, motivados principalmente por la consciencia de la importancia en la polinización de sus cultivos, se motivaron de rescatar saberes e incrementar las colmenas. Por lo que, a partir de sus innovaciones y liderazgo, otros productores se motivaban, los llamaron, se interesa-

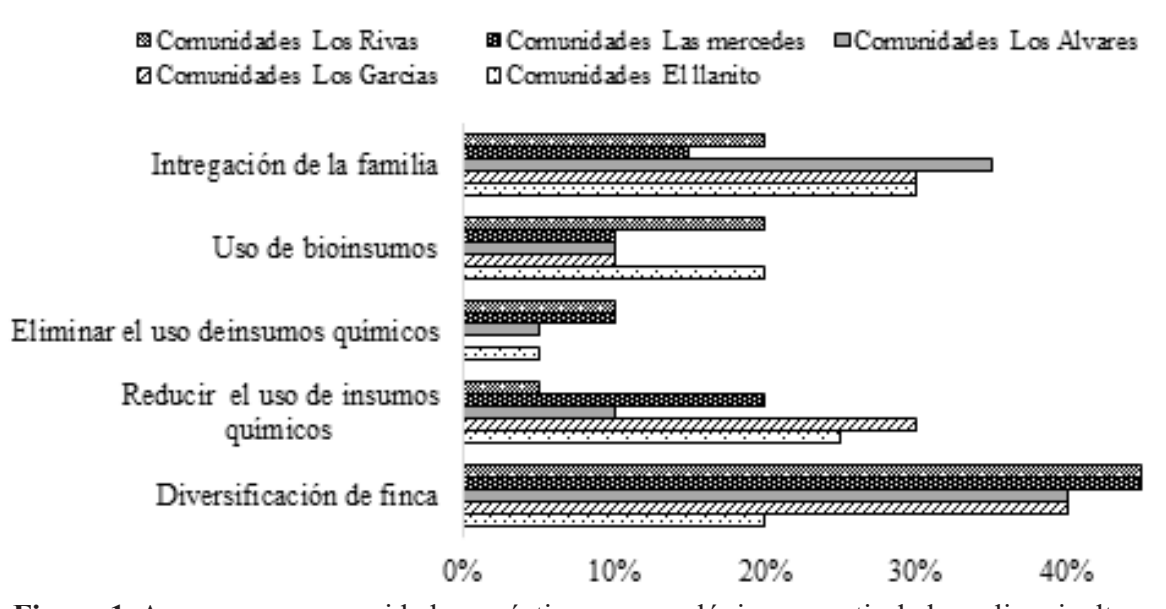

Figura 1. Avances por comunidad en prácticas agroecológicos a partir de la meliponicultura.
Para el rediseño de las fincas, las cinco comunidades, se articularon y realizaron dos viveros colectivos, con un total de 8000 plantas, 4000 cada uno, organizaron todo el proceso de forma colectiva, preparación de los sustratos, llenado de bolsa, sembrado, el riego, la resiembra, las actividades culturales, la colecta de semillas de frutales, maderables y melíferos, entre las especies sembradas fueron cacao, cítricos, pitahaya, níspero y otros. Se 
reunieron semanalmente a evaluar los avances, llevando control de los aportes individuales para la distribución equitativa de las plantas según el aporte de trabajo de cada cual. También, organizaron intercambios de semilla y material vegetativo, pitahaya, frijol canavalia, gandul, maíz, frijol, hortalizas, piña, coco, granadilla, hortalizas, granadilla, cálala, piña, coco y algunos árboles forestales.

En todo este proceso, la cultura local, el liderazgo y la fortaleza organizativa de los productores se fusionaron como un caldo de cultivo en la cotidianidad, de las actividades diarias, individuales y familiares. Siendo la base donde la actividad de rescate, crianza y producción de meliponinos realizada en interacción con promotores agroecológicos de avanzada fue tejiendo motivación, participación consciente y activa; responsabilidad y compromiso con la transición agroecológica de las propias fincas.

La cultura de los productores del municipio se caracteriza por la unidad, las comunidades están compuestas principalmente por una o dos familias, por lo cual la unidad es asociada a lazos familiares y a los liderazgos religiosos. Esta unidad se vincula con una fortaleza organizativa, redes territoriales de comunicación y apoyo, articulación y buena comunicación entre productores, técnicos institucionales y líderes locales.

La combinación de estos factores permitió que sin necesidad de proyectos externos o con alto financiamiento, se realizaran los intercambios, giras, y que las cajas y todo el manejo se realizara con recursos locales de los productores. Esto ha logrado que prevalezca una motivación auténtica en el rubro entre los productores (y no por pensar en algún beneficio material), que se retroalimenta continuamente con los intercambios, aprendizajes y evaluación de avances; y una mayor apropiación territorial, reconfiguración agroecológica del territorio y un nuevo rubro productivo en el municipio y dinamizar los procesos agroecológicos.

También fue relevante el liderazgo territorial del Técnico de Innovación del INTA en el municipio, que asume un rol de facilitador-organizador de los intercambios entre los productores, brindando acompañamiento y seguimiento a los acuerdos, realizando la mediación y resolución de conflictos, enlace con aliados externos municipales e intermunicipales y articulador de las fortalezas y recursos entre los productores.

En este proceso, las limitantes son las afectaciones de la mosca vinagrera; falta de rutas comerciales sólidas, la migración de productores jóvenes en ciertas partes del año hacia la ciudad o Costa Rica, que podría causar desequilibrios en los grupos e impactos organizativos.

\section{CONCLUSIONES}

La meliponicultura en el contexto histórico y social de Santa Lucia fue mediador pedagógico aprendizaje de la agroecología; junto a la interacción con los productores agroecológicos de mayor experiencia. Otros mediadores transversales fueron la cultura local, el liderazgo territorial y la fortaleza organizativa de los productores lo que ha permitido que las fincas están avanzando en la transición agroecológica, desde los recursos propios, un esfuerzo local para el desarrollo local y la sostenibilidad territorial.

Esta relación entre meliponinos y agroecología, es sumamente relevante en el contexto mundial de búsqueda de políticas públicas capaces de dinamizar los esfuerzos de escalonamiento agroecológico frente al cambio climático y la pobreza rural.

\section{REFERENCIAS BIBLIOGRÁFICAS}

Aldasoro, M. (2012). Documenting and Contextualizing Pjiekakjoo (Tlahuica) Knowledges thorugh a Collaborative Research Project. Recuperado de https://digital.lib.washington.edu/researchworks/bitstream/handle/1773/20792/AldasoroMaya_washington_0250E_10214.pdf?sequence=1\&isAllowed=y

Ayala, R., y Ortega, M. (2018). Declive de las abejas nativas en la región maya. Memorias del Primer coloquio de Meliponicultura: Liderazgo, Territorio y Tradición. 53-59. Recuperado de https://www.researchgate.net/profile/Ricardo_Ayala3/publication/330937889

Brosi, B. J., Daily, G. C., Shih, T. M., Oviedo, F., y Durán, G. (2008). The effects of forest fragmentation on bee communities in tropical countryside. Journal of Applied Ecology, 45(3), 773-783.

Castellanos, D., Castellanos, B., Silverio, M., Reinoso, C., y García, L. (2002). Aprender y enseñar en la escuela: Una concepción desarrolladora. La Habana, Cuba: Editorial Educación y Pueblo.

Chan, G., Anilú, G., Vera, G., Aldasoro, M., y Sotelo, L. (2019). Retomando saberes contemporáneos. Un análisis del panorama actual de la meliponicultura en Tabasco. Estudios de Cultura Maya, 53, 289-326.

Escobar, A. (2015). Territorios de diferencia: la ontología política de los derechos al territorio. Cuadernos de antropología social, (41), $25-38$.

INIDE (Insitituto Nicaraguense de Información de Desarrollo). (2008). Caracterización municipal. Santa Lucia en cifras. 
INTA (Instituto Nicaraguense de Tecnología Agropecuaria). (2019). Memorias I Encuentro Nacional de Meliponicultura y Apicultura.

Kjøhl, M., Nielsen, A., y Stenseth, N. C. (2011). Potential effects of climate change on crop pollination. Food and Agriculture Organization of the United Nations (FAO). Roma, Italia.

Genaro, J. A., y Lóriga, W. (2018). Melipona beecheii Bennett (Himenóptera: Apidae): origen, estudios y meliponicultura en Cuba. Insecta Mundi, 643, 1-18.

Guzmán, M., Balboa, C., Vandame, R., Albores, M. L., y González-Acereto, J. (2011). Manejo de las abejas nativas sin aguijón en México. Chiapas, México: El Colegio de la Frontera Sur.

Holt-Giménez, E. (2008). Campesino a Campesino: voces de Latinoamérica. Movimiento campesino para la agricultura sustentable. Managua, Nicaragua: SIMAS.

Lazos Chavero, E. (2009). La invención de los transgénicos: ¿Nuevas relaciones entre naturaleza y cultura?. Nueva antropología, 21(68), 9-35.

Luna, Y. (2012). Estrategias de Aprendizaje de Matemática en la Universidad Nacional Agraria de Nicaragua (Tesis de maestría). Universidad de la Habana. La Habana, Cuba.

Machín Sosa, B., Jaime, R., Lozano, Á., y Rosset, P. M. (2010). Revolución agroecológica: el movimiento de campesino a campesino de la ANAP en Cuba: Cuando el campesino ve, hace fe. ANAP: Habana, Cuba.

Meléndez, V., Ayala, R., Delfín, H., y Manrique, P. (2017). Diversidad de abejas en la reserva estatal de Dzilam. Recuperada de https://www.researchgate.net/publication/325828725_Diversidad_de_Abejas_de_la_Reserva

Mier, Y., Terán Giménez., Cacho, M., Giraldo, O. F., Aldasoro, M., Morales, H., Ferguson, B. G., Rosset, P., y Campos, C. (2018). Bringing agroecology to scale: Key drivers and emblematic cases. Agroecology and sustainable food systems, 42(6), 637-665.

Montenegro, J. (2013). Valoración del aporte de Meliponicultura a la economía familiar en: El Pochote, El Arenal y Nuevo Amanecer, comunidades del municipio de Masatepe, Masaya, 2013 (Tesis de pregrado). Recuperada de http://cenida.una.edu.ni/Tesis/tnl01m777. pdf

Perfecto, I., y Vandermer, J. (2012). Separación o integración para la conservación de la biodiversidad: la ideología detrás del debate "land sharing" frente a "land sapring". Ecosistema, 21(1-2), 180-191.

Rodríguez, N., McLaughlin, M., y Pennock, D. (2018). Soil pollution, a hidden reality. Organización de las Naciones Unidas para la Alimentación y Agricultura. Recuperado de http://www.fao.org/3/I9183EN/i9183en.pdf.

Sevilla, E. (2011). Sobre los orígenes de la agroecología en el pensamiento marxista y libertario. La paz, Bolivia: AGRUCO.

Talizina, N. (2000). Manual de Psicología Pedagógica. México: Universidad Autónoma de San Luis Potosí, México. 